\title{
Least Squares Technique for the Analysis of Periodic Temperatures of the Earth's Surface Region
}

\section{Tamami Kusuda}

\author{
Institute for Applied Technology, National Bureau of Standards, Washington, D.C. 20234
}

(October 19, 1966)

\begin{abstract}
A least squares technique has been applied to periodic earth temperature data for the purpose of determining basic characteristics of earth temperature cycles, such as thermal diffusivity, average temperature, amplitude, and phase angle of the temperature cycle. A new procedure was developed for obtaining a single thermal diffusivity which represents an average over time and depth at a particular temperature site. This thermal diffusivity was obtained as a nonlinear part of least squares constants which yielded a best-fit harmonic curve to a given set of observed earth temperatures. The thermal diffusivity thus calculated and the calculation method developed are preferred to those obtained by current practice, which yields two thermal diffusivities, one based on amplitude decay and another on phase angle shift.
\end{abstract}

Key Words: Earth temperature, least squares technique, thermal diffusivity of earth.

\section{Nomenclature}

$A_{0}, A_{1}, A_{2}, \ldots A_{N}=$ Least squares constants or best unbiased estimates of $a_{0}, a_{1}, a_{2}, \ldots a_{N}\left({ }^{\circ} \mathrm{C}\right)$

$a_{0}, a_{1}, a_{2}, \ldots a_{N}=$ Cosine coefficients of expected earth temperature function $\left({ }^{\circ} \mathrm{C}\right)$

$B_{1}, B_{2}, B_{3}, \ldots B_{N}=$ Least squares constants or best unbiased estimates of $b_{1}, b_{2}, b_{3}, \ldots . b_{N}\left({ }^{\circ} \mathrm{C}\right)$

$b_{1}, b_{2}, b_{3}, \ldots b_{N}=$ Sine coefficient of expected earth temperature function $\left({ }^{\circ} \mathrm{C}\right.$ )

$\hat{B}_{i}(x)=i$ th harmonic amplitude of earth temperature cycle at depth $x\left({ }^{\circ} \mathrm{C}\right)$

$C_{i}$ or $C_{i, K}=$ Linearized variables (dimensionless)

$D=$ A least squares estimate of average thermal diffusivity, $\alpha\left(\mathrm{cm}^{2} / \mathrm{sec}\right)$

$F(\theta)=$ Surface temperature function

$G_{i}=$ Amplitude of $i$ th harmonic coefficients $=\sqrt{a_{i}^{2}+b_{i}^{2}}$ $\left({ }^{\circ} \mathrm{C}\right)$

$M=$ Total number of temperature data (number of time data multiplied by number of depth data) (dimensionless)

$M_{t}=$ Total number of cycles to be considered (dimensionless)

$M_{x}=$ Total number of depths to be considered (dimensionless)

$N=$ Highest order of harmonics to be included for expected earth temperature function (dimensionless) $\hat{P}_{i}(x)=$ Phase angle of $i$ th harmonic of earth temperature cycle at depth $x$, (radians)

$S S=$ Sum of squares of all of the differences between observed and expected earth temperatures

$S_{i}$ or $S_{i, k}=$ Linearized variables (dimensionless)
$T=$ Period of cyclic data $(31,557,600 \mathrm{sec}$ for annual cycle)

$t=$ Earth temperature $\left({ }^{\circ} \mathrm{C}\right)$

$t_{\infty}=$ Earth temperature at $x \rightarrow \infty\left({ }^{\circ} \mathrm{C}\right)$

$t_{k}=$ Observed earth temperature $\left({ }^{\circ} \mathrm{C}\right)$

$x=$ Depth in earth $(\mathrm{cm})$

$x_{k}=$ Observed depth in earth $(\mathrm{cm})$

\section{Greek Characters}

$\alpha=$ Expected thermal diffusivity of earth $\left(\mathrm{cm}^{2} / \mathrm{sec}\right)$

$\beta_{i}=$ Temperature decay factor $=\sqrt{\frac{i \pi}{\alpha T}}\left(\mathrm{~cm}^{-1}\right)$

$\bar{\beta}_{i}=$ Estimate of $\beta_{i}=\sqrt{\frac{i \pi}{D T}}\left(\mathrm{~cm}^{-1}\right)$

$\delta=\frac{\partial S S}{\partial D}$

$\theta=$ Time coordinate used in the text, measured from January 1 (sec)

$\theta_{k}=$ Observed time with datum point at January 1 (sec) $\Phi_{i}=$ Phase angle of $i$ th harmonic of earth temperature,

(radians) $\Phi_{i}=\tan ^{-1} \frac{b_{i}}{a_{i}}$

$\Delta=$ Root mean squared deviation (defined in the text).

\section{Objective}

The purpose of this paper is to discuss a harmonic analysis of cyclic temperatures at selected depths of earth, The existing techniques employing a simple 
harmonic equation [Kusuda, 1965; Penrod, 1960] or Fourier analysis [Carson, 1963] have the disadvantage of yielding different thermal diffusivities for a given set of data, depending upon the choice of data reduction method, choice of cyclic period, choice of two depths, and the choice of computational method. The technique introduced in this paper eliminates this disadvantage and it is statistically more sound than previous methods for the analysis of periodic depth temperatures of earth. Furthermore, the method derived herein may be applied for the accurate calculation of thermal diffusivity of the surface layer of other celestial bodies in the event cyclic patterns of their depth temperatures are obtained in the future.

\section{Introduction and General Background}

Numerous investigators in the past have studied earth temperatures with reference to the earth's thermal diffusivity. A recent publication of Kusuda and Achenback [1965] includes an extensive bibliography on the subject. Most of the analyses in the papers listed in this bibliography apply the well-known periodic heat conduction formula for either diurnal or annual (or both) cycles of observed earth temperatures.

These analyses have been made to establish the average, the amplitude, and the phase angle of cyclic temperature patterns at various depth levels, so that earth temperatures may be predicted by a computational method. The amplitude decays and phase angle shifts of temperature cycles with respect to depths have been used to estimate the thermal diffusivity of the station where the earth observations were made. The analysis of periodic earth temperatures, therefore, eventually ends up with four basic parameters: thermal diffusivity, average temperature, amplitude, and phase angle of the surface temperature cycle from a given datum time point.

The investigators have encountered the following problems during the course of their studies. (1) The earth surface was difficult to define and earth surface temperatures were subject to disturbances from climatic changes above the surface, such as rainfall, cloud cover, evaporation of water, and wind velocity. (2) The actual earth temperatures observed did not follow an ideal simple-harmonic pattern. Moreover, their cyclic patterns varied from year to year and from day to day. Except in the work of Carson and in that of Kusuda and Achenbach, some degree of arbitrary human judgment was exercised to establish meaningful periodic patterns. For example, when several years of monthly average earth temperature records were available, the annual thermal diffusivity was calculated for each set of annual data. Such calculations imply an annual change of thermal diffusivity, which is a misapplication of basic heat conduction theory, as explained later. (3) For a given station, thermal diffusivities were computed both from the difference of logarithmic amplitudes and from the difference of phase angles for two different depths.
Unfortunately, the diffusivities calculated by these two methods usually did not agree, differing by a factor of two in some instances [Kusuda, 1965]. (4) When the thermal diffusivities were computed at two different pairs of two-depth levels at a given temperature station, two different estimates of the diffusivity were obtained, even when a consistent method of diffusivity computation was employed.

These four problems resulted in uncertainties of thermal diffusivities due to time, due to depth, due to method of computation, and due to the method of data reduction for a given set of earth conditions at a given locality.

Most of the investigators also attempted to predict earth temperatures based on an average of thermal diffusivities thus computed, and on other basic parameters; namely, the earth surface temperature amplitude, the phase angle of the earth surface temperature cycle measured from a certain reference datum point, and the average earth temperature during the cycle.

Mathematical description of an earth temperature cycle by a Fourier series technique, similar in nature with the least squares technique, has been tried by several authors. The most recent and comprehensive description was made by Carson [1963] for the soil of Lemont, Ill. The Fourier series technique is basically applicable only to a single complete cycle where data points consist of a unique set of temperatures for equally incremented time coordinates. Carson applied the technique to each individual one-year set of monthly average earth temperature cycles over a period of three years, resulting in three sets of Fourier coefficients, which did not agree with each other.

Kusuda and Achenbach [1965] applied a least squares technique in fitting observed earth temperature data to simple harmonic expressions for the purpose of computing the four basic parameters of earth temperature cycles.

In their least squares technique, Kusuda and Achenbach [1965] incorporated several years' records simultaneously, thus treating the temperature data as statistical variables or variates rather than functional variables, which is the case in a Fourier analysis. The least squares technique fits a sinusoidal curve to the observed data in such a manner that the variance calculated from the residual deviations of the observed temperature from the fitted curve is a minimum. The number of data points, the time intervals for the temperature observations, and the number of data for a given time are not restricted as they are in a Fourier analysis.

Kusuda and Achenbach [1965], however, began with the premise that the earth temperature cycles follow a simple harmonic mode. This premise may be reasonably applied to annual cycles of monthly average earth temperatures, but it is not quite valid for diurnal temperature cycles. They, however, still obtained two thermal diffusivities based upon, the amplitude decay and phase angle shift techniques, same as those used by other investigators.

The extension of the least squares technique to cover the higher harmonic terms is also desirable if the 
method is to be applicable to diurnal earth temperature cycles of the earth theoretically differing from the simple harmonic pattern because of a complex nonlinear surface heat exchange with the sun and the surrounding space.

In the following section the basic heat conduction relation of the periodic temperature cycle is first reviewed. The least squares technique is then applied to annual cycles of monthly average earth temperatures as an illustrative example.

\section{Basic Heat Conduction Theory on a Semi- Infinite Solid}

The surface layer of earth may be assumed to be a semi-infinite solid having a homogeneous thermal property, as far as the heat conduction theory is concerned. This assumption, however, may be inadequate in some cases, and a more refined model, such as composite solids (an upper layer of finite thickness over an infinitely thick solid bed of different thermal properties), is desirable. As a theory, this composite model probably represents more closely the actual surface of earth than the homogeneous model. But the thickness of the top layer, and the variation of properties between the top layer and the bottom bed are usually unknown, which makes the composite layer nodel less meaningful than the homogeneous model. Also, the mathematical complexity involved for the composite solid model may not be justified in finding an accurate thermal diffusivity of a surface layer from the observed depth temperature cycles. Hence, the homogeneous semi-infinite solid model is used to calculate average thermal diffusivity represented by the surface region of the earth. Or, conversely, temperature cycles at various depths are approximated by the equation derived for semi-infinite heat conduction models of homogeneous thermal properties.

The heat conduction of the semi-infinite homogeneous solid can be mathematically expressed as follows:

$$
\alpha \frac{\partial^{2} t}{\partial x^{2}}=\frac{\partial t}{\partial \theta}
$$

$$
\left.\begin{array}{lr}
\text { at } x \rightarrow \infty & t \rightarrow t_{\infty} \\
\text { at } x=0 & t=F(\theta) \\
\text { and } F(\theta+T)=F(\theta)
\end{array}\right]
$$

In actuality it is difficult to define a unique $F(\theta)$ that represents daily or annual cyclic observations of earth surface temperatures, since the character of these cycles varies from one period to the next. This variation is due to the varying nature of heat exchange over the earth surface with respect to climatic conditions. It is postulated that other celestial bodies without atmosphere may experience a much more consistent and stable $F(\theta)$ than the earth experiences. In this paper it is assumed that there exists a unique $F(\theta)$, which may be a statistical average of many periodic cycles. Since $F(\theta)$ is a periodic function of period $T$, it is possible to expand it as follows:

$$
F(\theta)=a_{0}+\sum_{i=1}^{\infty} a_{i} \cos \left(\frac{2 \pi i}{T} \theta\right)+\sum_{i=1}^{\infty} b_{i} \sin \left(\frac{2 \pi i}{T} \theta\right) .
$$

The values of $a_{0}, a_{1}, a_{2}, \ldots b_{1}, b_{2}, b_{3}$ are dependent upon the actual nature of the assumed surface temperature cyclic pattern.

The cyclic earth temperature [Eckert, 1959] at depth $x$ that satisfies (1), (2), and (3) is

$$
\begin{aligned}
t=t_{\infty}+\sum_{i=1}^{\infty} a_{i} e^{-\sqrt{\frac{i \pi}{\alpha T}} x} \cos \left(\frac{2 \pi i \theta}{T}-\sqrt{\frac{i \pi}{\alpha T}} x\right) \\
+\sum_{i=1}^{\infty} b_{i} e^{-\sqrt{\frac{i \pi}{\alpha T}} x} \sin \left(\frac{2 \pi i \theta}{T}-\sqrt{\frac{i \pi}{\alpha T}} x\right)
\end{aligned}
$$

(It should be noted from eqs (3) and (4) that $A_{0}=t_{\infty}$ ). Formula (4) can also be expressed as

$$
t=t_{\infty}+\sum_{i=1}^{\infty} G_{i} e-\sqrt{\frac{\sqrt{i \pi}}{\alpha T}} x \cos \left(\frac{2 \pi i \theta}{T}-\Phi_{i}-\sqrt{\frac{i \pi}{\alpha T}} x\right)
$$

where

$$
\begin{aligned}
& G_{i}=\sqrt{a_{i}^{2}+b_{i}^{2}} \\
& \tan \Phi_{i}=\frac{b_{i}}{a_{i} .}
\end{aligned}
$$

By letting

$$
\begin{aligned}
& G_{i} e^{-} \sqrt{\frac{i \pi}{\alpha T}} x=\hat{B}_{i}(x)=\text { depth amplitude } \\
& \Phi_{i}-\sqrt{\frac{i \pi}{\alpha T}} x=\hat{P}_{i}(x)=\text { depth phase angle, }
\end{aligned}
$$

the thermal diffusivity $\alpha$ can be readily calculated by knowing $\hat{B}_{i}(x)$ and $\hat{P}_{i}(x)$ at two different depths $x_{1}$ and $x_{2}$ by the following formulas:

$$
\alpha_{B i}=\frac{i \pi}{T}\left[\frac{x_{1}-x_{2}}{B_{i}\left(x_{2}\right)}\right]^{2}, \quad i=1,2 \ldots \infty
$$

and

$$
\alpha_{P i}=\frac{i \pi}{T}\left[\frac{x_{1}-x_{2}}{P_{i}\left(x_{1}\right)-\hat{P}_{i}\left(x_{2}\right)}\right]^{2}, \quad i=1,2 \ldots . \infty .
$$

In formulas (8) and (9), notation $\alpha_{B}$ and $\alpha_{P}$ are used instead of $\alpha$ to signify that these two thermal diffusivities are computed by amplitudes and by phase angles 
of $i$ th harmonic constants, respectively. Theoretically, of course

$$
\alpha_{B i}=\alpha_{P i}=\alpha \text { for } i=1,2,3, \ldots .
$$

Unfortunately, however, these relations have seldom been found in practice. Most previous researchers have made extensive studies on $\alpha_{B 1}$ and $\alpha_{P 1}$ only, ignoring higher harmonics. Even for the first harmonic constants, agreement between $\alpha_{B 1}$ and $\alpha_{P_{1}}$ was poor. Not only that, the values of $\alpha_{B 1}$ and $\alpha_{P 1}$ varied, as mentioned before, depending upon the choice of $x_{1}$ and $x_{2}$ of formulas (8) and (9). These difficulties have been chiefly attributed to the fact that the earth surface is not quite homogeneous and the heat conduction characteristics and surface temperature cycle of the earth surface region vary with respect to physical condition and time. The previous difficulties are, however, mostly due to the misuse of formula (4). It is not correct to use formula (4) either for analysis of one given periodic cycle of observation, or for analysis of depth variation of thermal diffusitivity. Instead, formula (4) should be used for the entire temperature data, covering the entire depth and the entire time period. For instance, if ten years of monthly average earth temperature cycles were complete with five depth readings, all of the 600 data points should be considered simultaneously for formula (4) to yield the best fit values of $t_{\infty},\left(a_{i}, i=1,2,3, \ldots\right)$ and $\left(b_{i, i=1,2}\right.$, 3 ...) as well as the best fit value of $\alpha$.

It is important here to mention that the foregoing statements are based upon the following statistical hypothesis:

Time data (including all the cyclic periods) follow basically a given harmonic function of a period $T$ (8766 hr for an annual earth cycle). Any deviation of actual cycles from a prescribed harmonic function are statistical rather than functional.

\section{The Least Squares Technique}

Although the technique described in this paper applies to any kind of periodic temperature data observed at several depths of any semi-solid system, the specific treatment will be given for annual cycles comprising monthly average earth temperatures taken at selected depths.

Assume that the number of annual cycles of earth temperature is $\mathrm{M}_{t}$ and each annual cycle consists of twelve monthly average readings at each of $M_{x}$ depths. If there are no missing data, although this is not a requirement, the total number of observations $M$ should be

$$
M=M_{t} \times M_{x} \times 12 .
$$

Equation (4), however, should be modified to include only the finite number of harmonic terms up to some larger number $N$. In other words, it is assumed that $a_{i}$ and $b_{i}$ will be numerically insignificant for all $i>N$.
The best unbiased estimates of $a_{0}, a_{1}, \ldots a_{N}$, $b_{1}, b_{2} \ldots b_{N}$, and $D$, that of $\alpha$ are given by the solutions of the following equations

$$
\begin{aligned}
& \frac{\partial S S}{\partial A_{0}}=0 \\
& \frac{\partial S S}{\partial A_{i}}=0 \\
& \frac{\partial S S}{\partial B_{i}}=0 \\
& \quad \delta=\frac{\partial S S}{\partial D}=0
\end{aligned}
$$

where $A_{0}, A_{1}, A_{2}, \ldots A_{N}, B_{1}, B_{2}, \ldots B_{N}$ and $D$ are the best estimates of $a_{0}, a_{1}, a_{2}, \ldots a_{N}, b_{0}, b_{1}, b_{2}, \ldots$ $b_{N}$, and $\alpha$.

$$
\text { And } \begin{aligned}
S S=\sum_{k=1}^{M} & {\left[t_{k}-A_{0}-\sum_{i=1}^{N} A_{i} e^{-\bar{\beta}_{i} x_{k}} \cos \left(\frac{2 \pi i}{T} \theta_{k}-\bar{\beta}_{i} x_{k}\right)\right.} \\
& \left.-\sum_{i=1}^{N} B_{i} e^{-\bar{\beta}_{i} x_{k}} \sin \left(\frac{2 \pi i}{T} \theta_{k}-\bar{\beta}_{i} x_{k}\right)\right]^{2}
\end{aligned}
$$

where

$$
\bar{\beta}_{i}=\sqrt{\frac{i \pi}{D T}}
$$

By letting

$$
\begin{gathered}
C_{i, k}=e^{-\bar{\beta}_{i} x_{k}} \cos \left(\frac{2 \pi i}{T} \theta_{k}-\bar{\beta}_{i} x_{k}\right) \\
S_{i, k}=e^{-\bar{\beta}_{i} x_{k}} \sin \left(\frac{2 \pi i}{T} \theta_{k}-\bar{\beta}_{i} x_{k}\right)
\end{gathered}
$$

and by noting that

$\frac{\partial S S}{\partial D}=0$ is also satisfied by $\frac{\partial S S}{\partial \beta_{i}}=0$ as long as $D \neq 0$,

the equations in (11) can be expressed simply by the linear matrix equation shown in table 1 . The lefthand side of the equal sign of table 1 is actually the product of two matrices, one square matrix of $(2 \mathrm{~N}$ $+1) \times(2 N+1)$ and one column matrix of dimension $(2 N+1)$. Equation $(11-a)$ is nonlinear and is expressed in terms of linearized variables, $C_{i, k}$ and $S_{i, k}$ as is shown in table 2 .

Determination of $A_{0}, A_{1}, A_{2}, \ldots A_{N}, B_{1}, B_{2}, \ldots$ $B_{N}$ as a best estimate of $a_{0}, a_{1}, a_{2}, \ldots a_{N}, b_{1}, b_{2}, \ldots$. $b_{N}$ is straightforward if the $C_{i, k}$ 's and $S_{i, k}$ 's are known. However, these variables include an unknown $D$ which is to be solved by $(11-a)$.

The method employed in this paper is to calculate a number of sets of $A_{0}, A_{i}, B_{i} ; i=1,2, \ldots N$, for several assumed $D$ 's. These calculated sets are then applied to the equation in table 2. By an iterative procedure, a set of $A_{0}, A_{i}, B_{i} ; i=1,2, \ldots N$, and $D$ 
Least Squares Equations

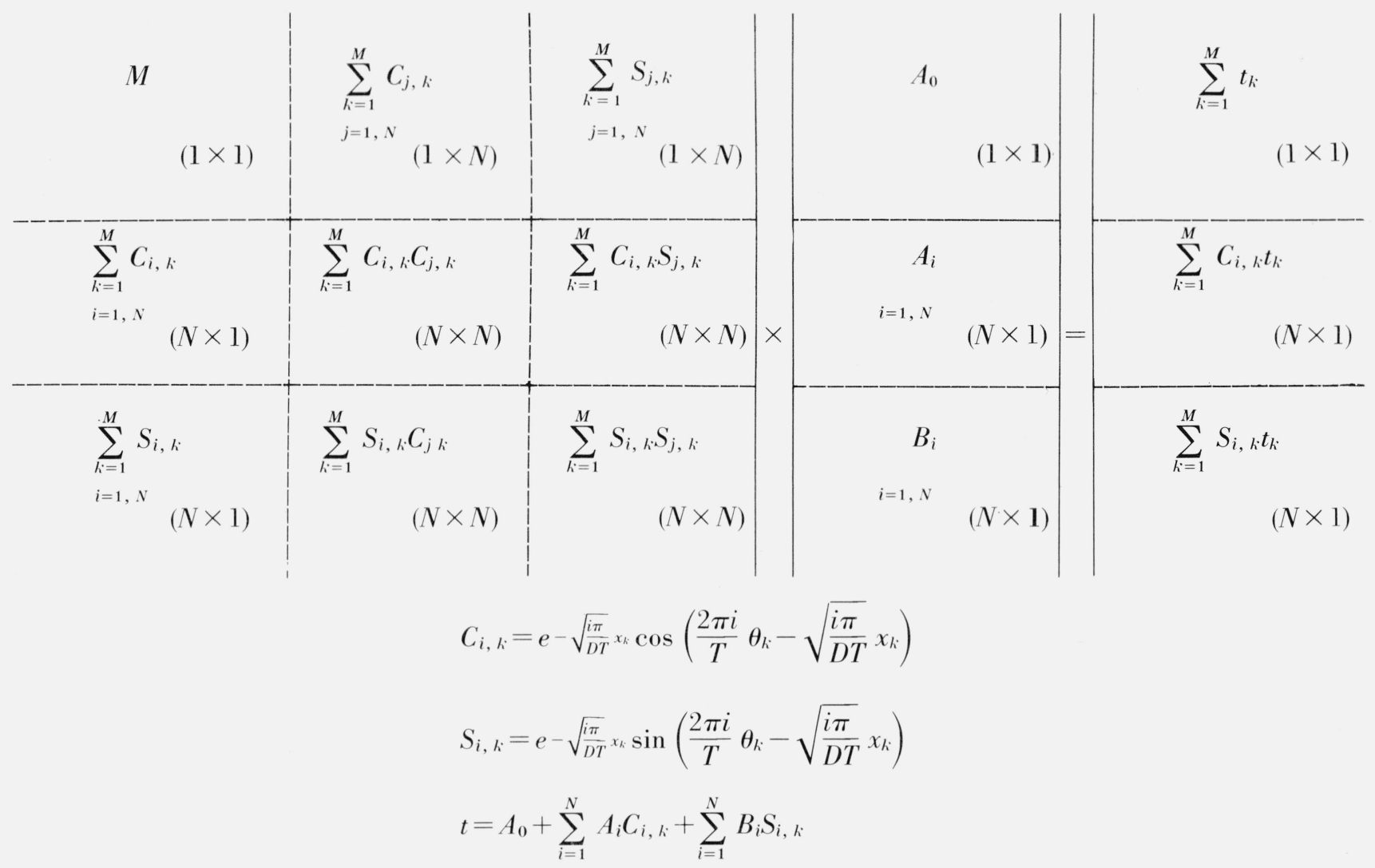

TABLE 2. Mathematical formula for the partial derivative of the sum of squares of all of the differences between observed and expected earth temperatures with respect to thermal diffusivity

$$
\begin{gathered}
\sum_{i}^{N} \sqrt{i(}\left(A_{i}+B_{i}\right) \sum_{k}^{M} t_{k} x_{k} C_{i, k}-\sum_{i}^{N} \sqrt{i}\left(A_{i}-B_{i}\right) \sum_{k}^{M} t_{k} \cdot x_{k} S_{i, k} \\
-A_{0} \sum_{i}^{N} \sqrt{i}\left(A_{i}+B_{i}\right) \sum_{h}^{M} x_{k} C_{i, k}+A_{0} \sum_{i}^{N} \sqrt{i}\left(A_{i}-B_{i}\right) \sum_{k}^{M} x_{k} S_{i, k} \\
-\sum_{i}^{N} A_{i} \sum_{j}^{N} \sqrt{j}\left(A_{j}+B_{j}\right) \sum_{k}^{M} C_{i, k} C_{j, k} x_{k}+\sum_{i}^{N} A_{i} \sum_{j}^{N} \sqrt{j}\left(A_{j}-B_{j}\right) \sum_{k}^{M} C_{i, k} S_{j, k} x_{k} \\
-\sum_{i}^{N} B_{i} \sum_{j}^{N} \sqrt{j}\left(A_{j}+B_{j}\right) \sum_{k}^{M} S_{i, k} C_{j, k} X_{k}+\sum_{i}^{N} B_{i} \sum_{j}^{N} \sqrt{j}\left(A_{j}-B_{j}\right) \sum_{k}^{M} S_{i, k} S_{j, k} x_{k} \\
=\delta=0
\end{gathered}
$$

can be found to satisfy $\delta=0$ (see table 2). These coefficients and the assumed $D$ are then substituted into formula (12) for the calculation of the mean squared deviation of the fitted least square values from the observed data by the following equation:

$$
\Delta^{2}=\frac{S S}{M} .
$$

The value of $\Delta$, or the square root of the average of the sum of squares of deviations is called hereafter the root mean squared deviations (RMSD) of the observed temperatures from those calculated by eq (4). The term "standard deviation" is purposely avoided here since the properties of these deviations resulting from the iterated nonlinear fitting procedure are not known. 
TABLE 3. Observed earth temperature data of Lemont, Ill., by Carson

\begin{tabular}{|c|c|c|c|c|c|c|c|}
\hline \multirow{2}{*}{ Month } & \multicolumn{7}{|c|}{ Depth $(\mathrm{cm})$} \\
\hline & 1 & 10 & 20 & 50 & 100 & 305 & 884 \\
\hline l & -0.4 & -0.2 & 0.2 & 1.8 & 4.0 & 9.7 & 11.3 \\
\hline 2 & -0.2 & -0.3 & 0. & 1.2 & 3.0 & 8.3 & 11.3 \\
\hline 3 & 4.0 & 3.3 & 3.0 & 2.9 & 3.5 & 7.3 & 11.4 \\
\hline 4 & 8.1 & 7.1 & 6.8 & 6.3 & 6.0 & 7.2 & \\
\hline 5 & 16.2 & 15.5 & 14.4 & 12.4 & 10.2 & 7.9 & \\
\hline 6 & 22.4 & 22.5 & 21.3 & 18.7 & 15.5 & 9.7 & \\
\hline 7 & 24.6 & 24.4 & 23.5 & 21.4 & 18.7 & 12.0 & 10.1 \\
\hline 8 & 24.0 & 23.7 & 23.0 & 21.7 & 19.8 & 13.8 & 10.1 \\
\hline 9 & 19.2 & 18.8 & 19.0 & 19.1 & 18.8 & 14.9 & 10.3 \\
\hline 10 & 14.1 & 14.2 & 14.6 & 15.3 & 15.9 & 14.8 & 10.4 \\
\hline 11 & 6.9 & 7.2 & 8.2 & 10.1 & 12.1 & 13.9 & 10.6 \\
\hline 12 & 1.8 & 1.9 & 2.8 & 4.8 & 7.8 & 12.5 & 11.0 \\
\hline 13 & -0.6 & -0.5 & 0.2 & 1.8 & 4.6 & 10.5 & 11.1 \\
\hline 14 & 1.1 & 0.8 & 0.9 & 1.8 & 3.5 & 8.9 & 11.2 \\
\hline 15 & 2.4 & 2.1 & 2.3 & 2.9 & 3.9 & 8.0 & 11.2 \\
\hline 16 & 10.7 & 10.1 & 9.3 & 7.9 & 6.7 & 7.5 & 11.0 \\
\hline 17 & 14.9 & 14.2 & 13.4 & 12.1 & 10.6 & 8.4 & 10.7 \\
\hline 18 & 23.6 & 22.9 & 21.4 & 18.6 & 15.4 & 10.0 & 10.6 \\
\hline 19 & 25.3 & 24.7 & 23.8 & 21.7 & 19.0 & 12.3 & 10.4 \\
\hline 20 & 23.9 & 23.2 & 22.8 & 21.4 & 19.7 & 14.2 & 10.5 \\
\hline 21 & 20.0 & 19.7 & 19.7 & 19.4 & 18.9 & 15.1 & 10.6 \\
\hline 22 & 13.5 & 13.3 & 14.0 & 15.2 & 16.1 & 15.2 & 10.9 \\
\hline 23 & 6.2 & 6.0 & 6.9 & 8.7 & 10.9 & 14.2 & 11.1 \\
\hline 24 & 1.3 & 1.1 & 1.9 & 4.0 & 7.2 & 12.3 & 11.3 \\
\hline 25 & 0.5 & 0.4 & 1.0 & 2.7 & 4.8 & 10.3 & 11.4 \\
\hline 26 & 0. & -0.4 & -0.1 & 1.2 & 3.4 & 9.0 & 11.5 \\
\hline 27 & 2.9 & 2.1 & 2.1 & 2.6 & 3.6 & 7.8 & 11.5 \\
\hline 28 & 11.3 & 11.0 & 10.2 & 8.6 & 7.1 & 7.4 & 11.1 \\
\hline 29 & 16.7 & 16.5 & 15.7 & 13.9 & 11.8 & 8.7 & 11.0 \\
\hline 30 & 20.6 & 20.1 & 19.3 & 17.4 & 15.0 & 10.4 & 10.7 \\
\hline 31 & 26.7 & 26.0 & 24.7 & 21.8 & 18.7 & 12.3 & 10.5 \\
\hline 32 & 25.5 & 25.1 & 24.4 & 22.9 & 20.7 & 14.2 & 10.5 \\
\hline 33 & 19.5 & 19.2 & 19.3 & 19.3 & 19.0 & 15.3 & 10.7 \\
\hline 34 & 13.3 & 13.1 & 13.6 & 14.7 & 15.7 & 15.3 & 10.8 \\
\hline 35 & 4.5 & 4.3 & 5.2 & 7.7 & 10.6 & 14.0 & 11.0 \\
\hline 36 & -0.3 & -0.4 & 0.4 & 2.7 & 5.8 & 12.1 & 11.4 \\
\hline
\end{tabular}

Sample Calculations: The normal equations of tables 1 and 2 have been solved for annual earth temperature data of Lexington, Ky., and Lemont, Ill., which had been obtained by Penrod [1960] and Carson [1963], respectively. The Kentucky data covered five years, from 1952 through 1956, at six depths, 0, 61, 122, 183, 244, and $305 \mathrm{~cm}$. Except for the data at 61 $\mathrm{cm}$ and $122 \mathrm{~cm}$ during July and August of 1953, the data are complete. Lemont data shown in table 3 are complete for the period of three years, from 1953 through 1955, at seven depths, 1, 10, 20, 50, 100, 305, and $884 \mathrm{~cm}$, except at the depth of $884 \mathrm{~cm}$ during May, June, and July of 1953.

Figures 1 and 2 illustrate the trend of $\delta$ (table 2) and corresponding values of $\Delta$ calculated for several values of thermal diffusivities for the earth temperature data of Carson and Penrod, respectively. The calculations in figures 1 and 2 were carried out for $N=4$.

It is seen in figure 2 that the value of $\Delta$, the RMSD of calculated temperatures from the observed data, decreases rapidly to the value of $1.07^{\circ} \mathrm{C}$ as the assumed thermal diffusivity increases to a value at 0.005 $\mathrm{cm}^{2} / \mathrm{sec}$. On the other hand it is seen from figure 2 that the diffusivity values that satisfy $\delta=0$ for the Lemont data is $\alpha=0.0058 \mathrm{~cm}^{2} / \mathrm{sec}$. Also it will be interesting to compare these thermal diffusivities with those calculated by conventional techniques by means of amplitude decay and phase angle shift by Penrod

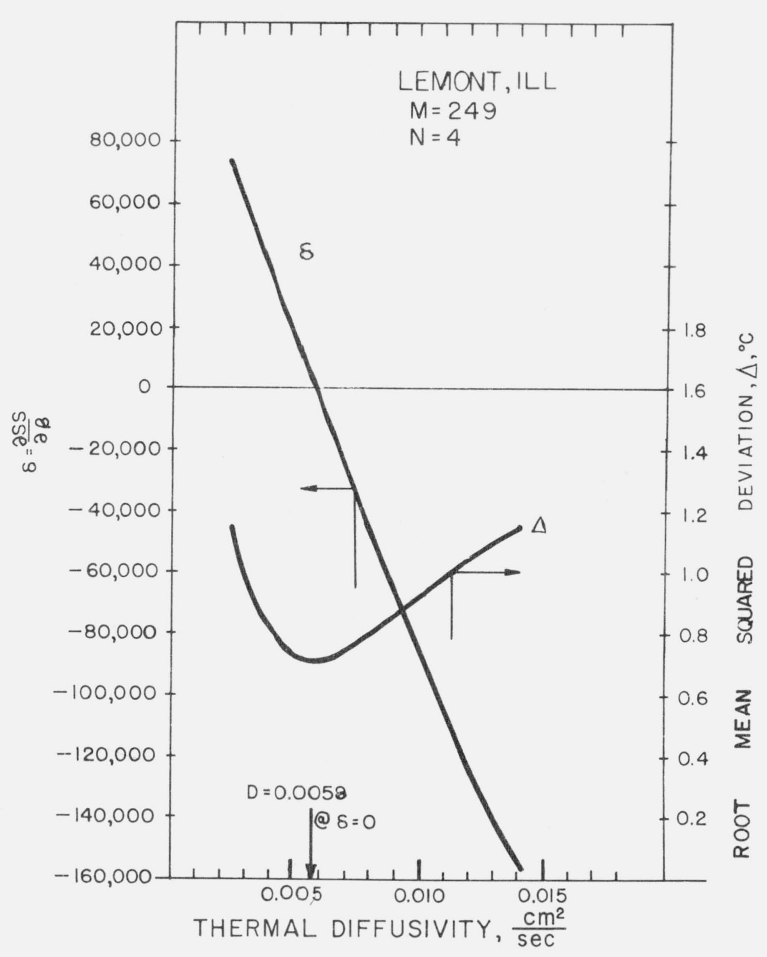

FIGURE 1. $\delta=\frac{\partial \mathrm{SS}}{\partial \beta}$ and $\Delta$ plotted against thermal diffusivity for earth temperature data of Lemont, Ill.

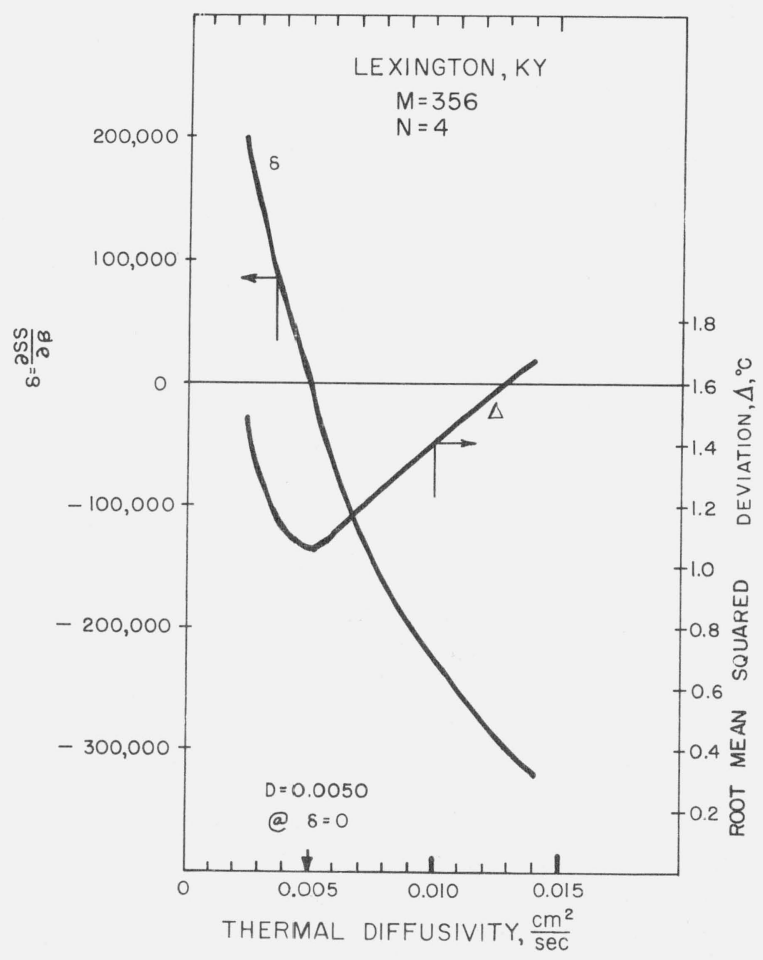

FIGURE 2. $\delta=\frac{\partial S S}{\partial \beta}$ and $\Delta$ plotted against thermal diffusivity for earth temperature data of Lexington, $K y$. 
from his Lexington data and with the data deduced from Carson's data for Lemont, Ill.

$$
\begin{aligned}
\text { Lexington data } \alpha_{B 1} & =0.0088 \mathrm{~cm}^{2} / \mathrm{sec} \\
\alpha_{P 1} & =0.0056 \mathrm{~cm}^{2} / \mathrm{sec} \\
\text { Lemont data } \alpha_{B 1} & =0.0048 \mathrm{~cm}^{2} / \mathrm{sec} \\
\alpha_{P 1} & =0.0045 \mathrm{~cm}^{2} / \mathrm{sec} .
\end{aligned}
$$

Aithough the thermal diffusivities calculated by conventional amplitude and phase angle techniques differ from one another considerably, the values of $\Delta$ corresponding to their diffusivities are all less than $1.3^{\circ} \mathrm{C}$ in their ranges, as seen in figures 1 and 2. (This small change of $\Delta$ with respect to a wide range of thermal diffusivity may be the very reason that the two methods can yield quite different thermal diffusivities.)

The mean squared deviation $\Delta^{2}$ may not be as insensitive to the thermal diffusivity for the other types of periodic temperature data as it has been found to be in the analysis on annual cycles of monthly average earth temperatures.

The Lexington earth temperature data were used to examine the influence of including higher harmonic terms on the values of $\Delta$ and it was found that the least squares analysis, including the higher harmonic terms up to degrees 2,4 , and 8 , do not yield values of $\Delta$ which are significantly different from the results of the analysis ignoring all of the higher harmonics for all the thermal diffusivities assumed.

Sample sets of least squares constants calculated for these two stations are shown in table 4.

TABLE 4. Least squares constants for earth temperature data of Lexington, Ky., and of Lemont, Ill.

\begin{tabular}{l|c|c}
\hline \hline & Lexington, Ky. & Lemont, Ill. \\
\cline { 2 - 3 }$M$ & 346 & 249 \\
$N$ & 4 & 4 \\
$D$ & 0.0050 & 0.0058 \\
$\Delta$ & 1.05 & .72 \\
$A_{0}$ & 14.48 & 11.39 \\
$A_{1}$ & -10.58 & -10.63 \\
$A_{2}$ & -0.30 & 0.08 \\
$A_{3}$ & -.54 & -.16 \\
$A_{4}$ & .24 & .30 \\
$B_{1}$ & -7.13 & -7.97 \\
$B_{2}$ & -0.38 & 0.59 \\
$B_{3}$ & 0.37 & .21 \\
$B_{4}$ & .14 & .39 \\
\hline
\end{tabular}

It may be interesting to compare the calculated earth temperatures based upon the least squares constants with the original observed data. Table 5 shows the calculated earth temperatures when $N=4$ is employed for formulas of table 1 on the observed data of Lemont, Ill. (table 3). Similar calculations such as those for table 4 for $N=1$ were also made to evaluate the improvement of taking higher harmonics over using the simple harmonic expression for the annual cycle of Lemont data. According to such comparison changes in the calculated results due to the inclusion of higher harmonic terms are very small. Table 5 also may be compared with table 6 , which is the arithmetic average (or norm) of the 3-year cyclic data of table 3. Significant discrepancy of the arithmetic average from the least squares result exists for the region near the surface.

TABLE 5. Predicted earth temperatures for Lemont, Illinois, using least squares constants up to fourth harmonics

\begin{tabular}{c|r|r|r|r|r|r|l}
\hline \multirow{3}{*}{ Month } & \multicolumn{7}{|c}{ Depth (cm) } \\
\cline { 2 - 7 } & \multicolumn{1}{|c|}{1} & 10 & 20 & 50 & 100 & 305 & 884 \\
\hline & & & & & & & \\
1 & -0.5 & -0.1 & 0.4 & 1.9 & 4.1 & 10.5 & 11.7 \\
2 & -0.3 & -0.0 & 0.3 & 1.4 & 3.1 & 9.0 & 11.7 \\
3 & 2.6 & 2.6 & 2.6 & 2.8 & 3.6 & 8.0 & 11.7 \\
4 & 9.4 & 9.0 & 8.6 & 7.6 & 6.7 & 7.7 & 11.6 \\
5 & 15.9 & 15.3 & 14.7 & 13.0 & 10.9 & 8.5 & 11.4 \\
6 & 21.8 & 21.1 & 20.3 & 18.2 & 15.4 & 10.0 & 11.3 \\
7 & 25.3 & 24.7 & 24.0 & 22.1 & 19.2 & 12.0 & 11.1 \\
8 & 23.7 & 23.5 & 23.2 & 22.3 & 20.5 & 13.9 & 11.0 \\
9 & 19.2 & 19.2 & 19.3 & 19.4 & 19.0 & 15.0 & 11.1 \\
10 & 13.2 & 13.6 & 14.0 & 15.0 & 15.9 & 15.1 & 11.2 \\
11 & 5.3 & 6.0 & 6.7 & 8.7 & 11.1 & 14.2 & 11.4 \\
12 & 0.5 & 1.1 & 1.8 & 3.7 & 6.6 & 12.5 & 11.5 \\
\hline
\end{tabular}

$M=249$.
$N=4$.

$D=.0058$.

TABLE 6. Arithmetic average earth temperature of Lemont, Illinois, earth temperature data in table 3

\begin{tabular}{c|r|r|r|r|r|r|l}
\hline \multirow{3}{*}{ Month } & \multicolumn{7}{|c}{ Depth (cm) } \\
\cline { 2 - 8 } & \multicolumn{1}{|c|}{1} & 10 & 20 & 50 & 100 & 305 & 884 \\
\hline & & & & & & & \\
1 & -0.2 & -0.1 & 0.5 & 2.1 & 4.4 & 6.8 & 11.3 \\
2 & .3 & .0 & .3 & 1.4 & 3.3 & 8.7 & 11.3 \\
3 & .9 & 3.1 & 2.5 & 2.8 & 3.7 & 7.1 & 11.4 \\
4 & 10.0 & 9.4 & 8.8 & 8.7 & 6.6 & 7.4 & 11.1 \\
5 & 15.9 & 15.4 & 14.5 & 12.8 & 10.9 & 8.3 & 10.8 \\
6 & 22.2 & 21.8 & 20.7 & 18.3 & 15.3 & 10.0 & 10.7 \\
7 & 25.5 & 25.1 & 24.0 & 21.6 & 18.8 & 12.2 & 10.3 \\
8 & 24.4 & 24.0 & 23.4 & 22.0 & 20.1 & 14.1 & 10.4 \\
9 & 19.6 & 19.2 & 19.3 & 19.3 & 18.9 & 16.1 & 10.6 \\
10 & 13.6 & 13.6 & 14.1 & 15.1 & 15.9 & 15.1 & 10.7 \\
11 & 5.9 & 5.8 & 6.8 & 8.8 & 11.2 & 14.1 & 10.9 \\
12 & 0.9 & 0.9 & 1.7 & 3.8 & 6.9 & 12.3 & 11.2 \\
\hline
\end{tabular}

Figure 3 compares the least squares calculation of the first harmonic constants with those derived from the observed three annual cycles of earth temperature in Lemont, Ill.

The coordinates $a_{x}$ and $b_{x}$ of the figure represent first harmonic constants describing the observed earth temperature at a depth $x$ from the ground surface. Since the Lemont data consists of seven depth observations for a period of three years, each depth point is indicated with three numerals representing the first, second, and third year.

The solid curve was obtained by connecting $a_{x}$ and $b_{x}$ calculated by theoretical relation (depicted in the upper right corner of fig. 3) for several depths. The calculations of $a_{x}$ and $b_{x}$ were made with the least squares constants indicated in table 5 . The curve representing the computed values of $a_{x}$ and $b_{x}$ fit extremely well for all of seven groups of three points. A similar comparison was made for the second harmonic constants of the same earth temperature data. 


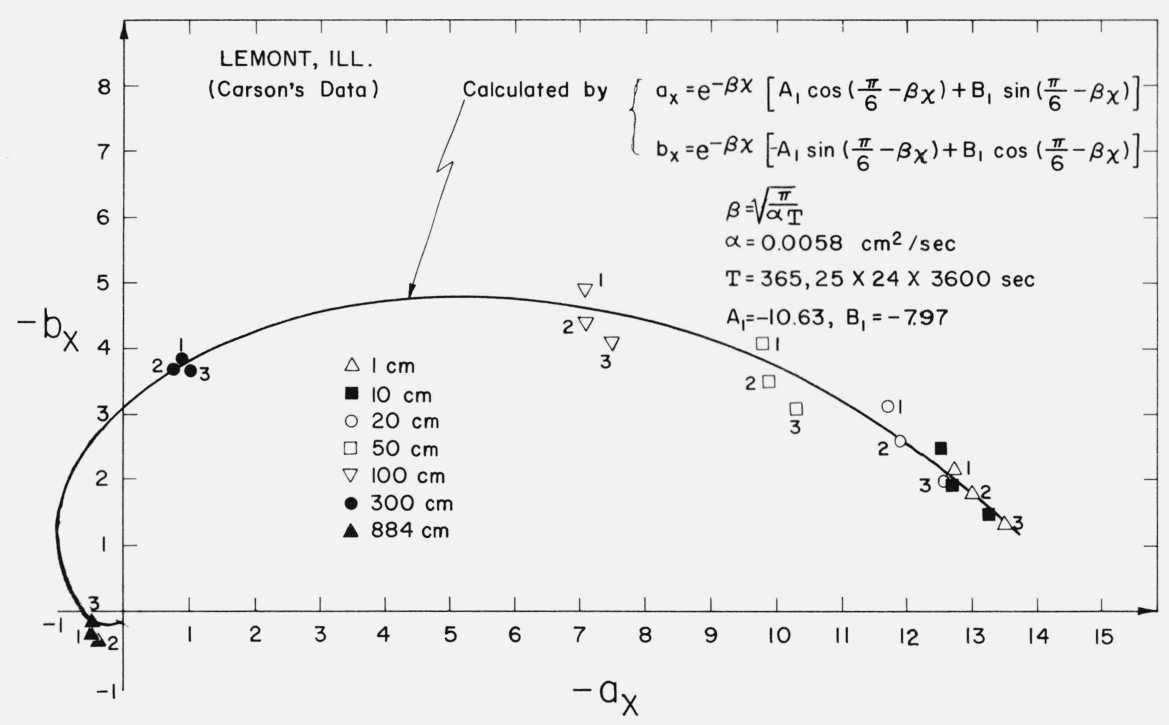

Figure 3. Graphical representation of harmonic constants for the earth temperature cycles of Lemont, Ill.

Although the scatter of these second harmonic constants derived from observed data is much more than those indicated in figure 3 , the computed curve did closely follow the centroid of the three points.

\section{Conclusions}

With the use of annual cyclic earth temperature data of Lexington, Ky., and of Lemont, Ill., a new technique is illustrated to yield single thermal diffusivity that will satisfy the least squares relation from the entirety of observed data. The technique requires an iterative solution of a nonlinear part of normal equations. The calculated earth temperatures based upon the theoretical heat conduction relation agrees very well with the average annual cyclic data of the monthly average earth temperatures when the least squares constants are properly evaluated.

It has also been found in this analysis that the deviation of the observed earth temperatures from the calculated temperatures is fairly insensitive to the thermal diffusivity as long as the thermal diffusivity is in the neighborhood of $0.005 \mathrm{~cm}^{2} / \mathrm{sec}$, at least for the annual cycles of monthly average earth temperatures for two stations studied in this paper. This insensitivity may be the very reason that the current and existing techniques resulted in two quite different thermal diffusivities depending upon the method of calculation used, the amplitude method or the phase angle method.
The method developed in this paper is recommended as the preferred method for the determination of thermal diffusivity from the periodic data of depth temperatures, not only of the earth surface by of other celestial bodies as well, in the event such data becomes available.

This paper is a modification of a report prepared under the contracted research for the Office of Civil Defense. The permission from OCD to release the paper for publication is appreciated.

The author greatly appreciates much critical review given by A. McNish of the National Bureau of Standards.

\section{References}

Carson, J. E., Analysis of soil and air temperature by Fourier technique, J. Geophys. Res. 68, No. 8, 2217-2232 (Apr. 15, 1963). Eckert, E. R. G., and Drake, R. M., Heat and Mass Transfer (McGrawHill Book Co., Inc., New York, N.Y.); Toronto, Canada; London, England, 99-107, 1959.

Kusuda, T., and Achenbach, P. R., Earth temperature and thermal diffusivity at selected stations in the United States, Transactions of American Society of Heating, Refrigerating and Air Conditioning Engineers 71, Pt. 1, 61-75 (1965).

Penrod, E. B., Variations of soil temperature at Lexington, Ky. from 1952 to 1956, Engineering Experiment Station Bulletin, University of Kentucky, No. 47 (Sept. 1960).

(Paper 71C1-442) 